\title{
Sesamol Inhibited Melanogenesis by Regulating Melanin-Related Signal Transduction in B16F10 Cells
}

\author{
Po-Yuan $\mathrm{Wu}^{1,2}{ }^{1}$, Ya-Jhen You ${ }^{3}$, Yi-Jung Liu ${ }^{3,4}$, Chien-Wei Hou ${ }^{5}$, Chin-Sheng $\mathrm{Wu}^{3}$, \\ Kuo-Ching Wen ${ }^{3}$, Chien-Yih Lin $^{6}$ and Hsiu-Mei Chiang ${ }^{3, *}$ \\ 1 Department of Dermatology, China Medical University Hospital, Taichung 40402, Taiwan; \\ wu.poyuan@gmail.com \\ 2 School of Medicine, China Medical University, Taichung 404, Taiwan \\ 3 Department of Cosmeceutics, China Medical University, Taichung 40402, Taiwan; \\ jenn123gay@yahoo.com.tw (Y.-J.Y.); ella8175@hotmail.com (Y.-J.L.); cswu@mail.cmu.edu.tw (C.-S.W.); \\ kcwen0520@mail.cmu.edu.tw (K.-C.W.) \\ 4 Ph.D Program for Biotechnology Industry, China Medical University, Taichung 40402, Taiwan \\ 5 Department of Biotechnology and Pharmaceutical Technology, Yuanpei University of Medical Technology, \\ Hsinchu 30015, Taiwan; rolis.hou@mail.ypu.edu.tw \\ 6 Department of Biotechnology, Asia University, Taichung 41354, Taiwan; yihlin@asia.edu.tw \\ * Correspondence: hmchiang@mail.cmu.edu.tw; Tel.: +886-4-22053366 (ext. 5302); Fax: +886-4-22078083
}

Received: 23 January 2018; Accepted: 3 April 2018; Published: 7 April 2018

\begin{abstract}
Melanin is synthesized through a series of interactions catalyzed by melanogenic enzymes such as tyrosinase, dopachrome tautomerase (tyrosinase-related protein-2; TRP-2), and tyrosinase-related protein-1 (TRP-1). Tyrosinase plays a key role in catalysing the initial and limiting steps of melanogenesis. The melanin that results from melanogenesis has the protective effect of absorbing ultraviolet radiation. However, overproduction of melanin, in addition to altering the appearance of skin, may lead to skin disorders such as melasma, solar lentigo, and postinflammatory hyperpigmentation. Previous studies have revealed that sesamol is a strong antioxidant and a free radical scavenger. In this study, we investigated the effects of sesamol on the regulation of melanogenesis and related mechanisms in B16F10 cells. The results indicated that sesamol inhibited tyrosinase activity and melanogenesis induced by $\alpha$-melanocyte-stimulating hormone $(\alpha-\mathrm{MSH})$ in B16F10 melanoma cells. Sesamol decreased the protein level of melanocortin 1 receptor (MC1R), microphthalmia-associated transcription factor (MITF), tyrosinase, and TRP-1 by downregulating cyclic adenosine monophosphate (cAMP)/protein kinase A (PKA) pathways that had been activated by $\alpha$-MSH. Sesamol increased glycogen synthase kinase 3 beta (GSK3 $\beta)$, protein kinase B (AKT), and extracellular signal-related kinase (ERK) phosphorylation, thus inhibiting the transcription of MITF. Sesamol also inhibited melanin synthesis and tyrosinase expression by modulating ERK, phosphoinositide 3-kinase (PI3K)/AKT, p38, and c-Jun amino-terminal kinase (JNK) signalling pathways. These results indicate that sesamol acted as a potent depigmenting agent.
\end{abstract}

Keywords: sesamol; melanogenesis; tyrosinase; microphthalmia-associated transcription factor (MITF); melanocortin 1 receptor (MC1R); glycogen synthase kinase 3 beta (GSK3 $\beta$ )

\section{Introduction}

Skin colour is the manifestation of the progressive transfer of melanin to keratinocytes after melanogenesis in the melanosomes of melanocytes. Long-term ultraviolet (UV) exposure causes an abnormal increase in reactive oxygen species (ROS) generation, which induces melanogenesis to protect skin from the deleterious effects of UV irradiation and environmental pollutants [1-3]. However, excessive accumulation of melanin can influence appearance and cause pigmentation 
disorders such as freckles, age spots, solar lentigo, postinflammatory hyperpigmentation, melasma, and even melanoma [4,5].

Melanin synthesis comprises a series of complex processes regulated by various factors, including enzymes, proteins and hormones [6,7]. The limiting enzyme in melanogenesis is tyrosinase. L-tyrosine is transported from the extracellular space or intracellular generation through hydroxylation of L-phenylalanine, a precursor of tyrosine. Hydroxylation of tyrosine by tyrosinase produces L-3,4-dihydroxyphenylalanine (L-DOPA), which subsequently is oxidized to DOPAquinone [8]. DOPAquinone undergoes nonenzymatic intramolecular cyclization reaction to form leucochrome and then nonenzymatically oxidized to DOPAchrome. Tyrosinase-related protein-2 (TRP-2) will convert DOPAchrome to 5,6-dihydroxyindole-2-carboxylic acid (DHICA), which is catalysed by TRP-1 to form eulmelanin [9-11]. TRP-1 and TRP-2 stabilize and increase the activity of tyrosinase. Tyrosine and L-DOPA serve as substrates and intermediates of melanogenesis. In addition, they also act as inducers and positive regulators of the melanogenic pathway and of other cellular functions [12]. The cells surrounding melanocytes such as keratinocytes and fibroblasts may influence melanogenesis. $\alpha$-Melanocyte-stimulating hormone $(\alpha-\mathrm{MSH})$ is a product of the processing of proopiomelanocortin (POMC), which, along with adrenocorticotropic hormone (ACTH), is produced in a regulated fashion by all resident skin cells, including keratinocytes, melanocytes, and fibroblasts, as well as by immune cells $[7,13] . \alpha$-MSH binds to the melanocortin 1 receptor (MC1R) on melanocytes. Cyclic adenosine monophosphate (cAMP) stimulates the translocation of protein kinase A (PKA) into the nucleus, thus activating cAMP-response element-binding protein (CREB) $[8,14]$. Activation of cAMP increases microphthalmia-associated transcription factor (MITF) expression, which upregulates tyrosinase, TRP-1, and TRP-2 expression, ultimately promoting melanin synthesis in melanocytes [15].

Agents with antioxidant and tyrosinase inhibition activity can be used to prevent hyperpigmentation $[15,16]$. Sesame seeds contain strong antioxidants and are common food in Central and East Asia. Sesamol (3,4-(methylenedioxy)phenol), an active component in sesame seeds, is a potent antioxidant which scavenges free radicals $[17,18]$. Liu et al. have demonstrated that sesamol inhibits proliferation and promotes apoptosis of HepG2 cells [19]. Sesamol was also reported to prevent cardiovascular disease, coronary heart disease, and stroke [20]. The present study aimed to investigate the effect of sesamol on melanin synthesis in B16F10 cells. Sesamol's effect on the regulation of cAMP/PKA, mitogen-activated protein kinase kinase (MEK)/extracellular signal-related kinase (ERK), protein kinase B (AKT)/glycogen synthase kinase 3 beta (GSK3 $\beta) / C R E B$, TRP-1, and MITF in melanin synthesis were also studied.

\section{Results}

\subsection{B16F10 Cell Viability with Sesamol Treatment}

After treatment with 10-200 $\mu \mathrm{M}$ sesamol for $48 \mathrm{~h}$, cell viability was found to be higher than the $80 \%$ viability found at $10-50 \mu \mathrm{M}$. However, cell viability was $66.1 \%$ and $55.7 \%$ with 100 and $200 \mu \mathrm{M}$ of sesamol treatment, respectively (Figure 1). Thus, the doses of sesamol used to study its effect on melanogenesis were $10-50 \mu \mathrm{M}$. 


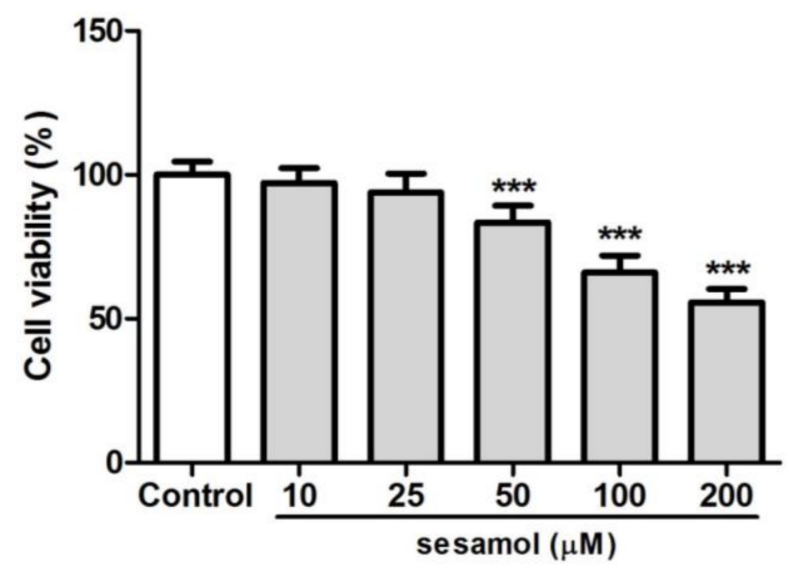

Figure 1. Cell viability (\%) of B16F10 cells after $48 \mathrm{~h}$ of treatment with sesamol. The cell viability was below $80 \%$ at sesamol doses over $100 \mu \mathrm{M}$. Each value is presented as the mean $\pm \mathrm{SD}$. Significant difference with control group: ${ }^{* * *} p<0.001$.

\subsection{Sesamol Inhibited Melanin Biosynthesis in B16F10 Cells}

Figure 2 shows the effects of sesamol on melanin content in B16F10 cells. The intracellular melanin content increased to $191.9 \% \pm 3.5 \%$ after $\alpha$-MSH treatment. Sesamol at doses higher than $5.0 \mu \mathrm{M}$ significantly reduced the melanin content. After $50 \mu \mathrm{M}$ sesamol treatment, melanin decreased to $90.1 \%$ $\pm 3.3 \%$ (Figure 2). The cell pellet was darker after $\alpha$-MSH treatment, but it became lighter in the sesamol group. According to the results, sesamol significantly inhibited melanin biosynthesis.

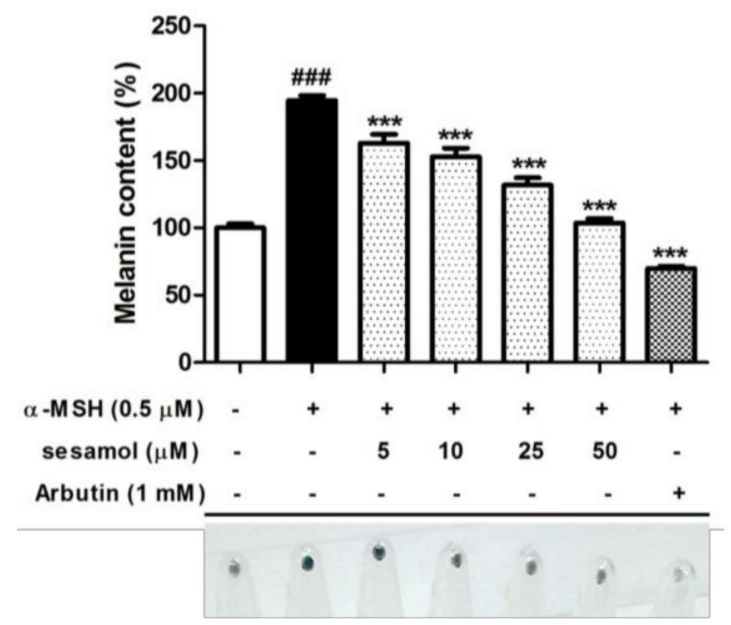

Figure 2. Melanin content (\%) of B16F10 cells and cell pellets after $48 \mathrm{~h}$ of treatment with sesamol. Seasamol significantly inhibited melanin synthesis. Each value is presented as the mean \pm SD. Significant difference versus control: ${ }^{\# \#} p<0.001$. Significant difference versus $\alpha$-MSH-treated group: *** $p<0.001$. Positive control: $1 \mathrm{mM}$ arbutin.

\subsection{Sesamol Inhibited Tyrosinase Activity in B16F10 Cells}

Tyrosinase is the rate-limiting enzyme in melanin synthesis. Inhibition of tyrosinase activity is an efficient strategy in developing of antimelanogenic agents. Sesamol significantly inhibited tyrosinase activity in B16F10 cells (Figure 3A). The levels of tyrosinase activity were $159.6 \% \pm 1.0 \%$ after $\alpha$-MSH treatment, and became $154.9 \% \pm 3.2 \%, 146.7 \% \pm 5.7 \%, 141.3 \% \pm 2.3 \%$, and $133.6 \% \pm 6.4 \%$ after treatment with $5,10,25$, and $50 \mu \mathrm{M}$ sesamol, respectively. The results indicated that sesamol inhibited tyrosinase activity in B16F10 cells. 


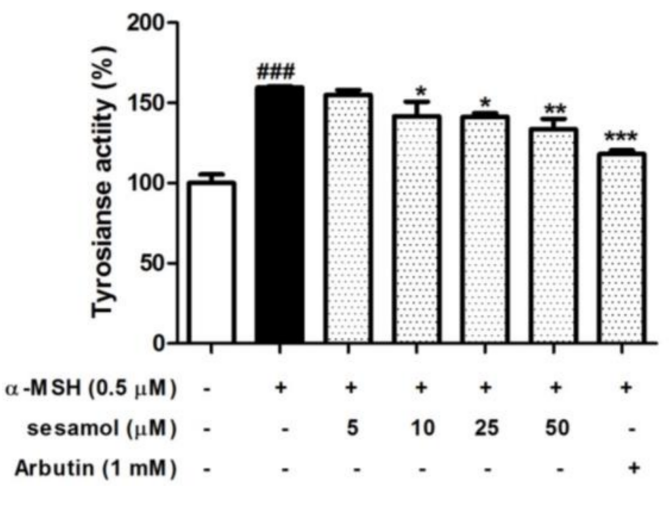

(A)
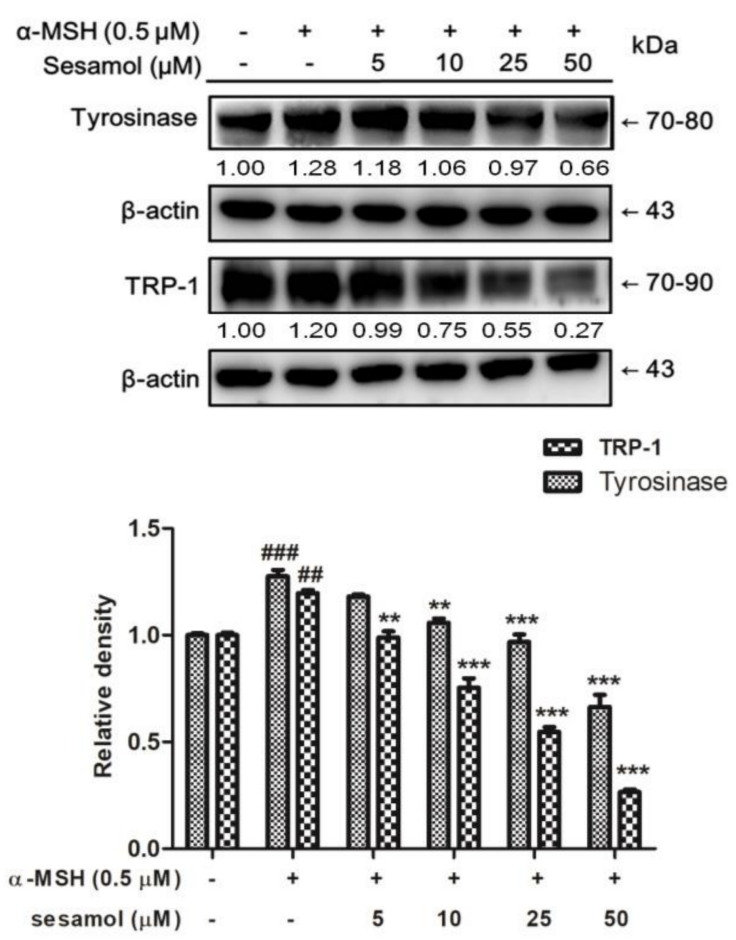

(B)

Figure 3. (A) Tyrosinase activity (\%) of B16F10 cells after $48 \mathrm{~h}$ of treatment with sesamol. Sesamol inhibited tyrosinase activity in B16F10 cells. Each value is presented as the mean \pm SD. Significant difference versus control: ${ }^{\# \#} p<0.001$. Significant difference versus $\alpha$-MSH-treated group: ${ }^{*} p<0.05$, ${ }^{* *} p<0.01,{ }^{* * *} p<0.001$. Positive control: $1 \mathrm{mM}$ arbutin. (B) Effect of sesamol on $\alpha$-MSH-induced protein expression of tyrosinase and TRP-1 in B16F10 cells. Sesamol suppressed tyrosinase and TRP-1 protein levels. Each value is presented as the mean \pm SD. Significant difference versus control: ${ }^{\# \#} p<0.001$, $\#$ \# $p<0.01$. Significant difference versus $\alpha$-MSH-treated group:** $p<0.01,{ }^{* * *} p<0.001$.

\subsection{Sesamol Inhibited Tyrosinase and TRP-1 Protein Expression in B16F10 Cells}

In a protein expression assay using Western blotting, tyrosinase expression increased to 1.28 -fold in the $\alpha$-MSH group, and the protein expression of tyrosinase decreased to 1.18-, 1.06-, 0.97-, and 0.66-fold of the control value after 5-50 $\mu \mathrm{M}$ sesamol treatment for $48 \mathrm{~h}$. Sesamol suppression of tyrosinase expression was dose dependent (Figure 3B). The results show that sesamol inhibited melanogenesis in B16F10 cells by suppressing tyrosinase activity and protein expression.

To understand the mechanism underlying sesamol's regulatory effect on melanogenesis, the protein expression of TRP- 1 was determined in B16F10 cells after their treatment with $\alpha$-MSH and $5-50 \mu \mathrm{M}$ sesamol for $48 \mathrm{~h}$. The results show that at doses higher than $5 \mu \mathrm{M}$, sesamol significantly reduced the TRP-1 level (Figure 3B).

\subsection{Sesamol Downregulated MC1R and MITF Expression}

MC1R expresses in melanocytes and is a key receptor in melanogenesis [21]. The expression of MC1R was found to be 1.10-fold for the control value. MC1R expression was 1.16-, 1.00-, 0.96-, and 0.69 -fold with 5-50 $\mu \mathrm{M}$ sesamol treatments for $2 \mathrm{~h}$ (Figure 4). With $50 \mu \mathrm{M}$ sesamol treatment, the protein expression of MC1R was significantly less than at the control value. MITF expression in B16F10 cells increased to 1.88 -fold of the control in the $\alpha$-MSH group (Figure 4). Inhibition of MITF expression varied with the sesamol treatment dose; at a concentration of $25 \mu \mathrm{M}$ sesamol, MITF expression in the B16F10 cells was significantly downregulated (Figure 4). 

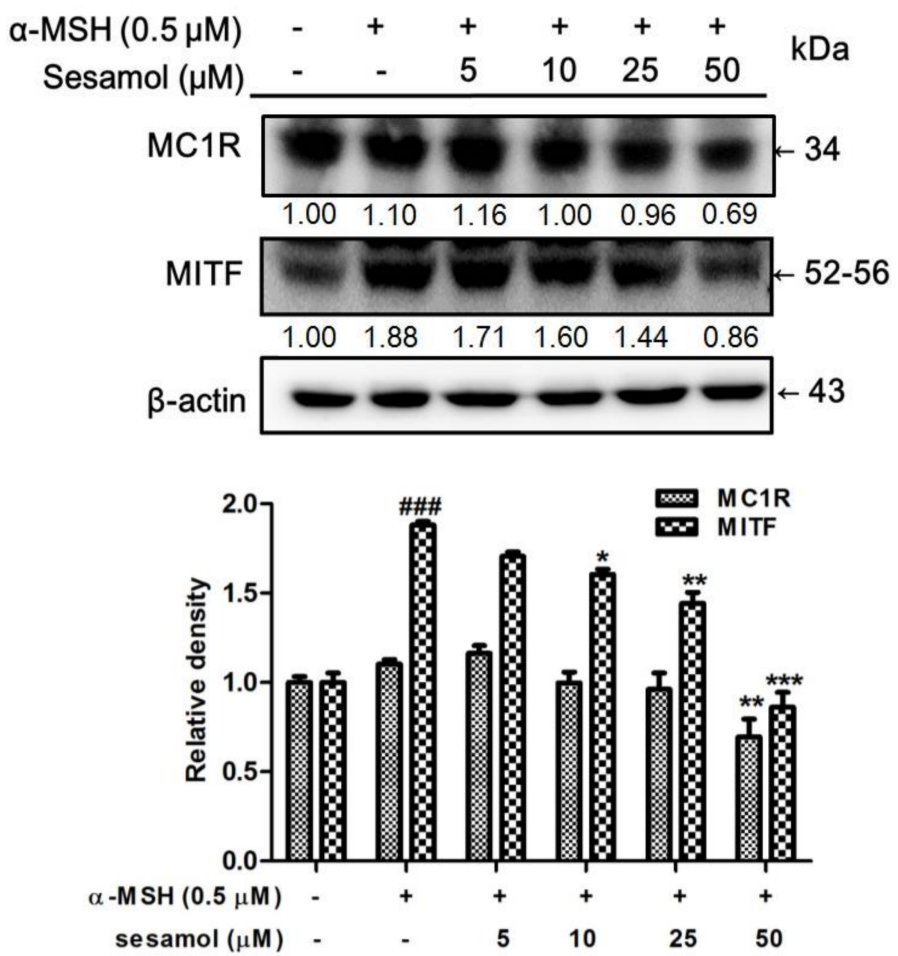

Figure 4. Effect of sesamol on $\alpha$-MSH-induced protein expression of MC1R and MITF in B16F10 cells. Each value is presented as the mean \pm SD. Significant difference versus control: ${ }^{\# \#} p<0.001$; Significant difference versus $\alpha$-MSH-treated group: ${ }^{*} p<0.05,{ }^{* *} p<0.01,{ }^{* * *} p<0.001$.

\subsection{Sesamol Inhibited Melanogenesis by Upregulating $p-A K T$ and $p$-GSK3 $\beta$ Expression}

Inhibition of the phosphorylation of AKT and GSK3 $\beta$ leads to the MITF activation resulting in melanin synthesis [22]. The expressions of AKT and GSK3 $\beta$ were determined to clarify the regulation of sesamol in this pathway. As shown in Figure 5, treatment with $\alpha$-MSH significantly decreased $p$-AKT (0.24-fold of control) and $p$-GSK3 $\beta$ (0.65-fold of control) expression in B16F10 cells. Treatment with $10 \mu \mathrm{M}$ sesamol for $48 \mathrm{~h}$ markedly increased $p$-AKT expression, and $48 \mathrm{~h}$ treatment with $5 \mu \mathrm{M}$ of sesamol markedly increased $p$-GSK3 $\beta$ expression. These results indicate that sesamol activated the phosphorylation of AKT and GSK3 $\beta$, leading to the downregulation of downstream signalling transduction, such as MITF expression, and resulting in inhibition of tyrosinase gene activity and expression, and, therefore, melanin synthesis.

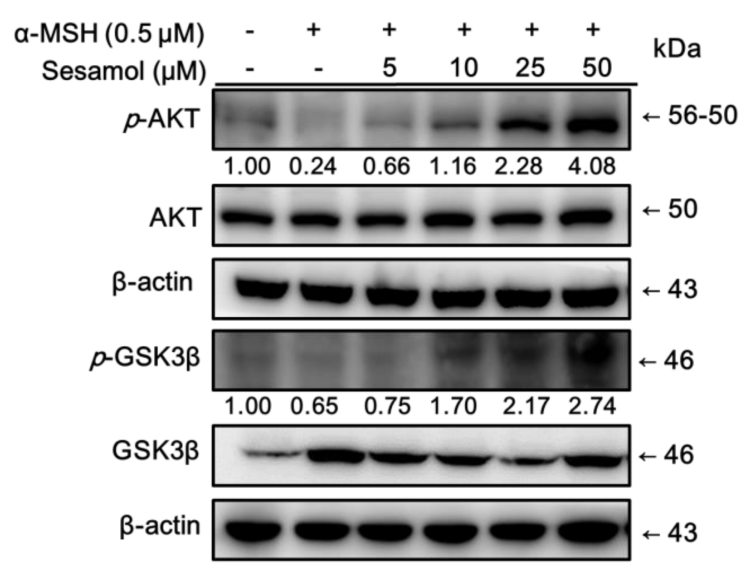

Figure 5. Cont. 


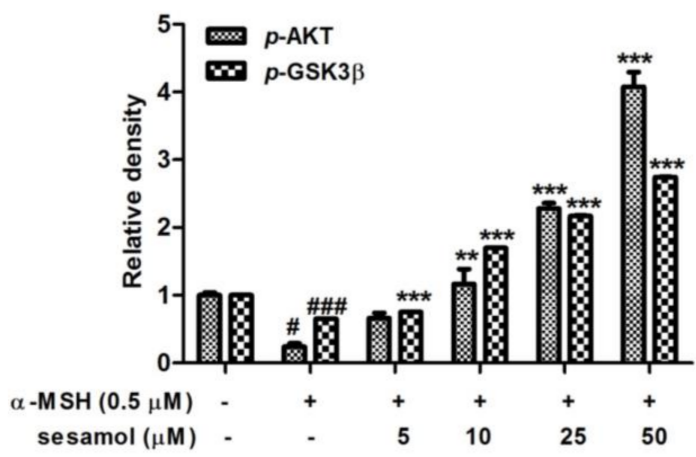

Figure 5. Effect of sesamol on $\alpha$-MSH-induced protein expression of $p$-GSK3 $\beta$ and $p$-AKT in B16F10 cells. Each value is presented, in comparison with the control group, as the mean $\pm \mathrm{SD} .{ }^{\#} p<0.05$, and ${ }^{\# \# \#} p<0.001$; and, in comparison with the $\alpha-\mathrm{MSH}$ treated group, as ${ }^{* *} p<0.01$, and ${ }^{* *} p<0.001$.

\subsection{Sesamol Upregulated p-ERK Expression}

Phosphorylation of ERK activates MITF to stimulus melanin synthesis. To understand the role of ERK in antimelanogenesis of sesamol, the expression of ERK was studied. After treatment with $\alpha$-MSH, $p$-ERK expression in B16F10 cells became 0.82-fold compared with $p$-ERK expression in the control (Figure 6). Sesamol dose-dependently inhibited $p$-ERK expression in B16F10 cells after $48 \mathrm{~h}$ of treatment.

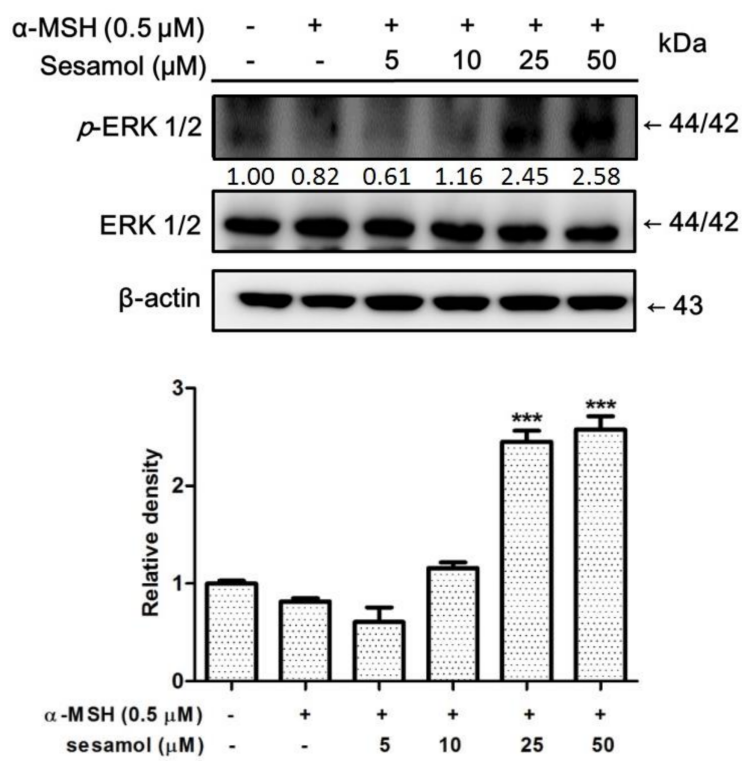

Figure 6. Effect of sesamol on $\alpha$-MSH-induced protein expression of $p$-ERK in B16F10 cells. Each value is presented, in comparison with the $\alpha$-MSH-treated group, as the mean $\pm \mathrm{SD}$. Significant difference versus $\alpha$-MSH-treated group: ${ }^{* * *} p<0.001$.

\subsection{Effects of Sesamol on the Melanogenesis Signalling Pathway}

To understand the mechanism underlying the depigmenting effect, we examined sesamol in cAMP/PKA, PI3K/AKT, MEK/ERK, p38, and JNK pathways along with H-89 (PKA/mitogen- and stress-activated protein kinase (MSK) inhibitor), LY 294002 (PI3K inhibitor), PD 98059 (ERK inhibitor), SB 203580 (p38 inhibitor), and JNK inhibitor II, respectively, for $48 \mathrm{~h}$.

\subsubsection{Inhibition of Melanogenesis by Sesamol was Associated with PKA/MSK Regulation}

To determine whether inhibition of melanogenesis by sesamol was associated with the PKA/MSK pathway, B16F10 cells were incubated with $10 \mu \mathrm{M} \mathrm{H}-89$ and $50 \mu \mathrm{M}$ sesamol for $48 \mathrm{~h}$. The melanin 
content and expression of tyrosinase was determined. As Figure 7A shows, the melanin content of B16F10 cells increased to $149.4 \% \pm 4.9 \%$ relative to the control upon treatment with $\alpha-\mathrm{MSH}$. Subsequently, separate treatments with sesamol and $\mathrm{H}-89$ reduced $\alpha-\mathrm{MSH}$-induced melanin content to $101.4 \% \pm 4.5 \%$ and $81.5 \% \pm 6.9 \%$ compared with that in the control, respectively (Figure 7A). In addition, cotreatment with sesamol and $\mathrm{H}-89$ reduced melanin content to $65.7 \% \pm 1.5 \%$ compared with that in the control.

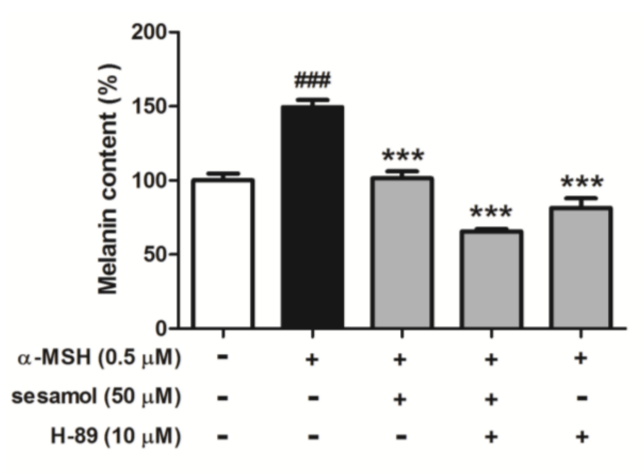

(A)

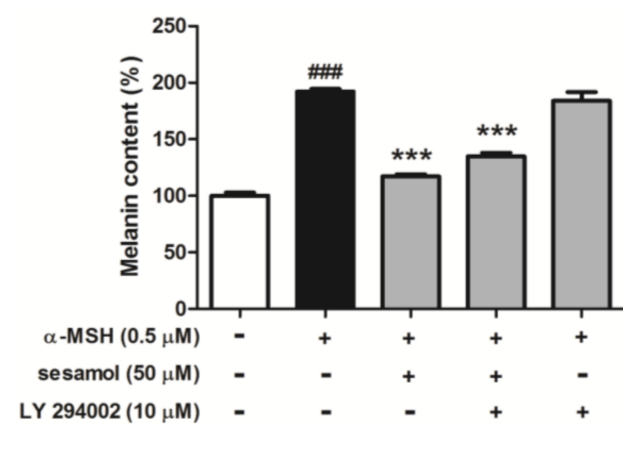

(C)
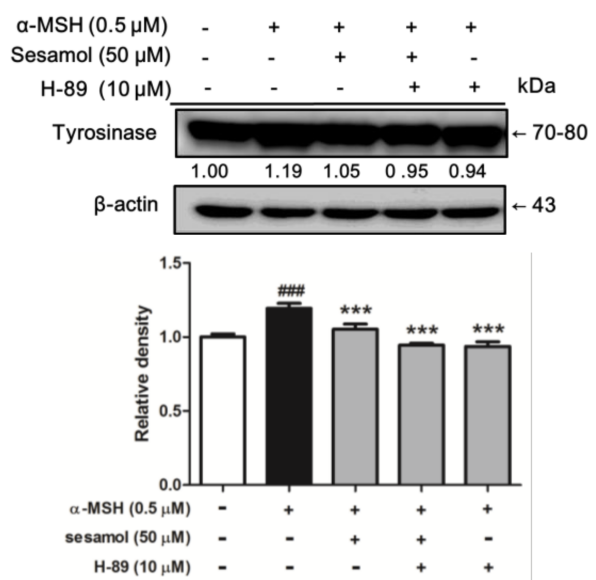

(B)

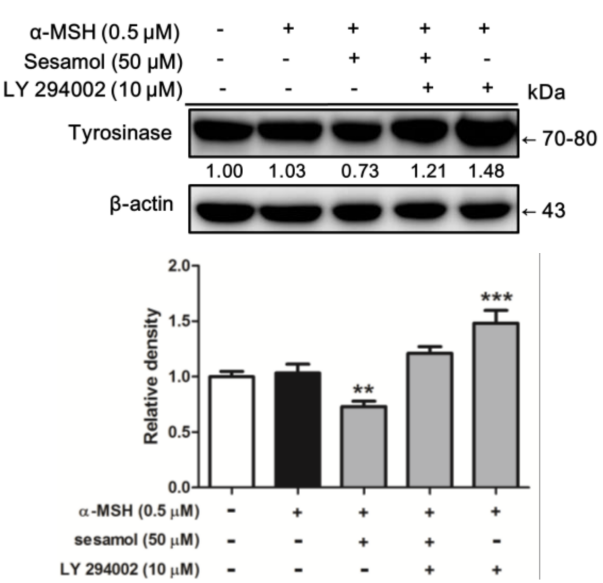

(D)

Figure 7. (A) Effect of sesamol and H-89 (PKA/MSK inhibitor) on melanin content (\%) in $\alpha$-MSH-treated B16F10 cells after $48 \mathrm{~h}$. Each value is presented as the mean $\pm \mathrm{SD}$. \#\# $p<0.001$ (Significant difference versus control); ${ }^{* *} p<0.001$ (Significant difference versus $\alpha$-MSH treated group). (B) Effects of sesamol and H-89 (PKA/MSK inhibitor) on protein expression of tyrosinase in $\alpha$-MSH-treated B16F10 cells after $48 \mathrm{~h}$. Each value is presented as the mean \pm SD. ${ }^{\# \#} p<0.001$ (Significant difference versus control); ${ }^{* * *} p<0.001$ (Significant difference versus $\alpha$-MSH treated group). (C) Effects of sesamol and LY 294002 (PI3K inhibitor) on melanin content (\%) in $\alpha$-MSH-treated B16F10 cells after $48 \mathrm{~h}$. Each value is presented as the mean $\pm \mathrm{SD}$. ${ }^{\# \#} p<0.001$ (Significant difference versus control); ${ }^{* *} p<0.001$ (Significant difference versus $\alpha$-MSH treated group). (D) Effects of sesamol and LY 294002 (PI3K inhibitor) on protein expression of tyrosinase in $\alpha$-MSH-treated B16F10 cells after $48 \mathrm{~h}$. Each value is presented as the mean $\pm \mathrm{SD} .{ }^{* *} p<0.01,{ }^{* * *} p<0.001$ (Significant difference versus $\alpha$-MSH treated group).

As shown in Figure 7B, tyrosinase expression was 1.19-fold of the control after $\alpha$-MSH treatment, but decreased to 1.05- and 0.94-fold, respectively, after sesamol and H-89 treatments. In addition, after cotreatment with sesamol and H-89, tyrosinase expression in B16F10 cells decreased to 0.95-fold 
of that in the control. These results indicate that PKA and MSK pathways may be involved in the antimelanogenic effect of sesamol.

\subsubsection{Sesamol Inhibited Melanogenesis by Inhibiting PI3K}

To determine whether inhibition of melanogenesis by sesamol was regulated by the PI3K pathway, B16F10 cells were incubated with $10 \mu \mathrm{M}$ LY 294002 and $50 \mu \mathrm{M}$ sesamol for $48 \mathrm{~h}$. The melanin content and expression of tyrosinase was determined. The melanin content of B16F10 cells increased to $192.3 \% \pm 2.2 \%$ after $\alpha$-MSH treatment, while separate incubation with sesamol and LY 294002 (PI3K inhibitor) reduced $\alpha$-MSH-induced melanin content to $117.1 \% \pm 1.9 \%$ and $183.9 \% \pm 7.8 \%$, respectively (Figure 7C). In addition, cotreatment with sesamol and LY 294002 reduced melanin content to $134.8 \% \pm 3.1 \%$ compared with that in the control.

As shown in Figure 7D, tyrosinase expression was 1.03-fold of the control after $\alpha$-MSH treatment, but 0.73- and 1.48-fold, respectively, after separate sesamol and LY 294002 treatments. In addition, cotreatment with sesamol and LY 294002 decreased tyrosinase expression to 1.21-fold of that in the control. The results indicate that the PI3K pathway may be involved in the antimelanogenic effect of sesamol.

\subsubsection{Sesamol Inhibited Melanogenesis by Inhibiting ERK}

To determine whether inhibition of melanogenesis by sesamol was regulated by ERK, B16F10 cells were incubated with $10 \mu \mathrm{M}$ PD 98059 and $50 \mu \mathrm{M}$ sesamol for $48 \mathrm{~h}$. The melanin content and expression of tyrosinase was determined. The melanin content of B16F10 cells increased to $199.2 \% \pm 0.6 \%$ after $\alpha$-MSH treatment. Separate incubation with sesamol and $10 \mu \mathrm{M}$ PD 98059 (ERK inhibitor) subsequently altered $\alpha$-MSH-induced melanin content to $90.6 \% \pm 1.6 \%$ and $246.2 \% \pm 7.0 \%$, respectively, compared with that in the control (Figure 8A). In addition, after cotreatment with sesamol and PD 98059, melanin content increased to $105.7 \% \pm 1.4 \%$ compared with that in the control.

As shown in Figure 8B, the tyrosinase expression was 1.35-fold of that in the control after $\alpha$-MSH treatment, but after separate sesamol and PD 98059 treatments, tyrosinase expression was 0.97- and 1.77-fold of the control, respectively. In addition, cotreatment with sesamol and PD 98059 increased tyrosinase expression to 1.05-fold of the control. The results indicate that the ERK pathway may be involved in the antimelanogenic effect of sesamol.

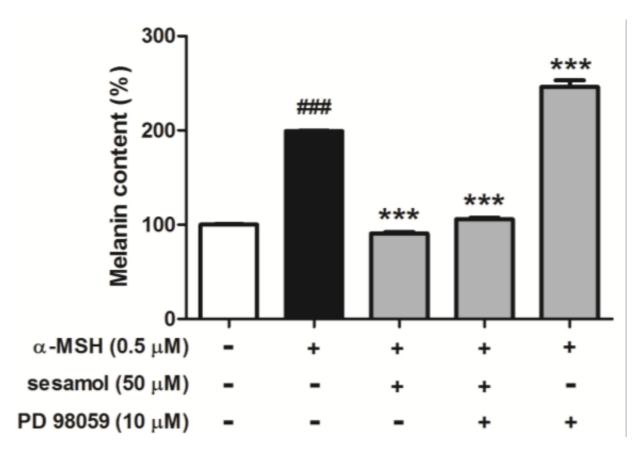

(A)

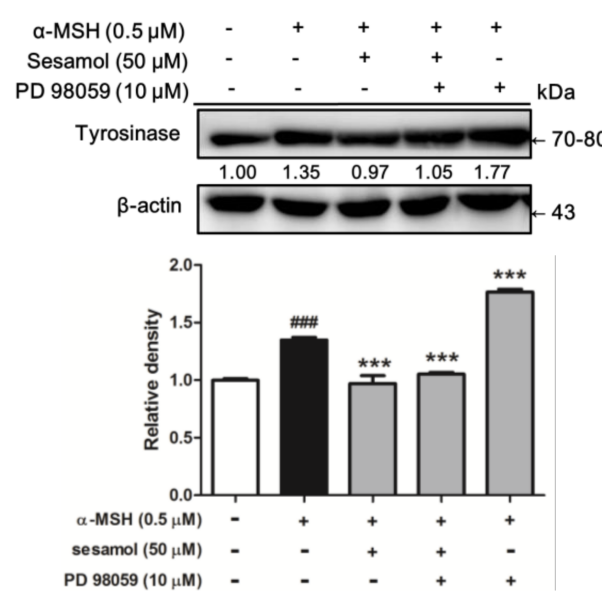

(B)

Figure 8. (A) Effects of sesamol and PD 98059 (ERK inhibitor) on melanin content (\%) in $\alpha$-MSH-treated B16F10 cells after $48 \mathrm{~h}$. Each value is presented as the mean \pm SD. ${ }^{\# \#} p<0.001$ (Significant difference versus control); ${ }^{* * *} p<0.001$ (Significant difference versus $\alpha$-MSH treated group). (B) Effects of sesamol and PD 98059 (ERK inhibitor) on protein expression of tyrosinase in $\alpha$-MSH-treated B16F10 cells after $48 \mathrm{~h}$. Each value is presented as the mean \pm SD. ${ }^{\# \#} p<0.001$ (Significant difference versus control); *** $p<0.001$ (Significant difference versus $\alpha$-MSH treated group). 


\subsubsection{Sesamol Inhibited Melanogenesis by Inhibiting p38}

To determine whether inhibition of melanogenesis by sesamol was regulated by p38, B16F10 cells were incubated with $10 \mu \mathrm{M}$ SB 203580 and $50 \mu \mathrm{M}$ sesamol for $48 \mathrm{~h}$. The melanin content of B16F10 cells increased to $176.6 \% \pm 11.4 \%$ after $\alpha$-MSH treatment, while separate incubation with sesamol and $10 \mu \mathrm{M}$ SB 203580 (p38 inhibitor) regulated $\alpha$-MSH-induced melanin content to $129.2 \% \pm 5.4 \%$ and $235.0 \% \pm 4.8 \%$, respectively, compared with that in the control (Figure 9). In addition, after cotreatment with sesamol and SB 203580, melanin content was $168.2 \% \pm 12.6 \%$ compared with that in the control.

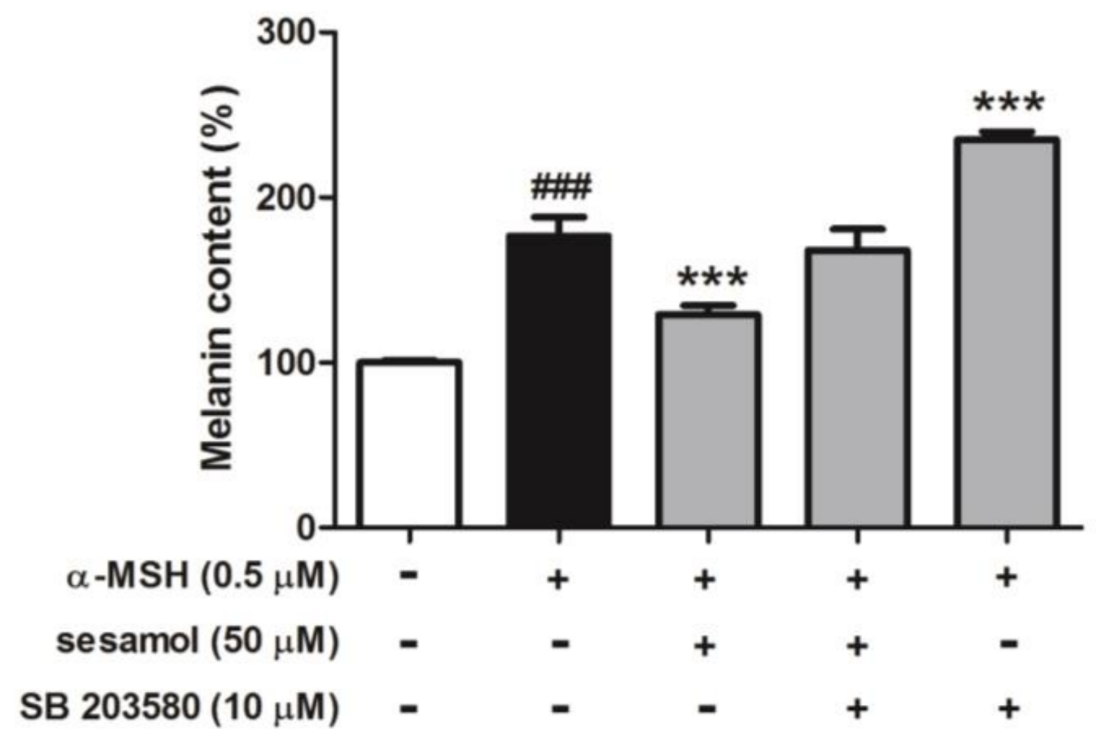

Figure 9. Effects of sesamol and SB 203580 (p38 inhibitor) on melanin content (\%) in $\alpha$-MSH-treated B16F10 cells after $48 \mathrm{~h}$. Each value is presented as the mean \pm S.D. ${ }^{\# \#} p<0.001$ (Significant difference versus control); ${ }^{* * *} p<0.001$ (Significant difference versus $\alpha$-MSH treated group).

\subsubsection{Sesamol Inhibited Melanogenesis by Inhibiting JNK}

To determine whether inhibition of melanogenesis by sesamol was regulated by JNK, B16F10 cells were incubated with $10 \mu \mathrm{M}$ JNK inhibitor II and $50 \mu \mathrm{M}$ sesamol for $48 \mathrm{~h}$. The melanin content and expression of tyrosinase was determined. The melanin content of B16F10 cells increased to $186.6 \% \pm 3.6 \%$ after $\alpha$-MSH treatment, while incubation with sesamol and $10 \mu \mathrm{M}$ JNK inhibitor II separately regulated $\alpha$-MSH-induced melanin content to $95.5 \% \pm 8.0 \%$ and $197.1 \% \pm 1.2 \%$ compared with that in the control, respectively (Figure 10A). In addition, after cotreatment with sesamol and JNK inhibitor II, melanin content was $116.7 \% \pm 2.4 \%$ compared with that in the control.

Figure 10B shows that tyrosinase expression was 1.20 -fold of the control value after $\alpha$-MSH treatment, but the protein expression was 0.47- and 0.74-fold after separate sesamol and JNK inhibitor II treatment. In addition, cotreatment with sesamol and JNK inhibitor II increased tyrosinase expression to 0.54 -fold of the control. The results indicate that the JNK pathway may be involved in the antimelanogenic effect of sesamol. 


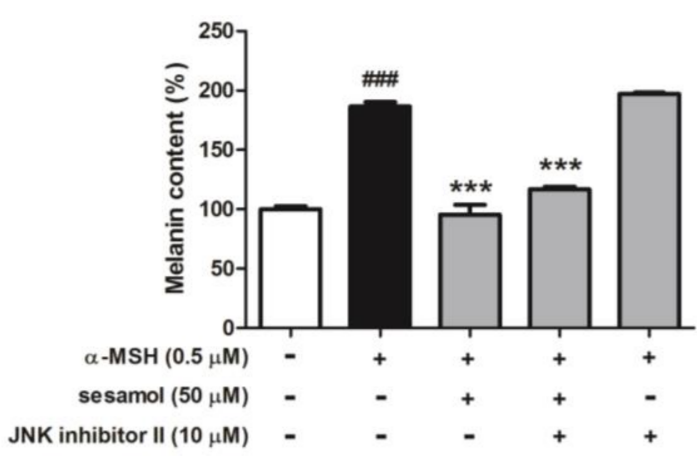

(A)

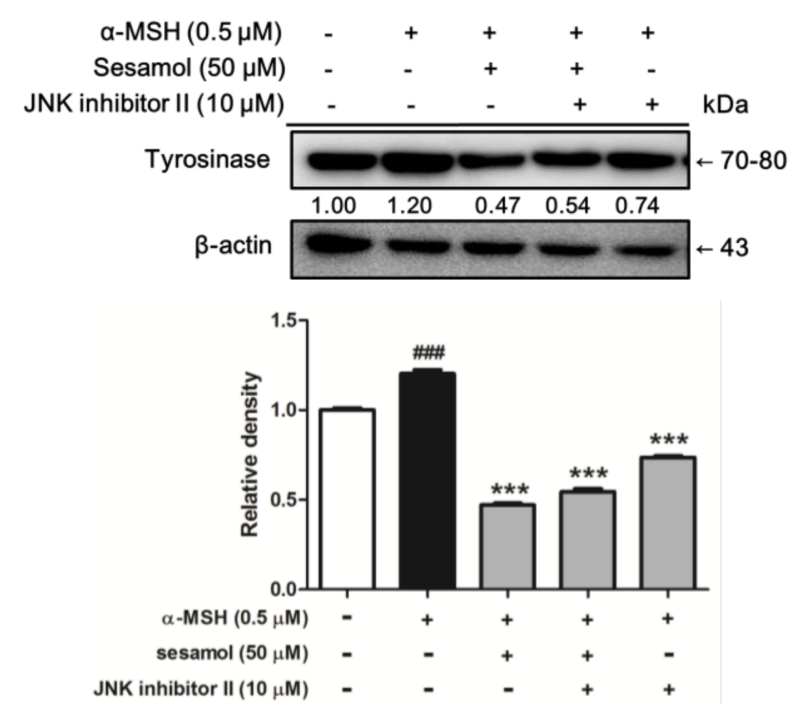

(B)

Figure 10. (A) Effect of sesamol and JNK inhibitor II on melanin content (\%) in $\alpha$-MSH-treated B16F10 cells after $48 \mathrm{~h}$. Each value is presented as the mean \pm SD. ${ }^{\# \#} p<0.001$ (Significant difference versus control); ${ }^{* *} p<0.001$ (Significant difference versus $\alpha$-MSH treated group). (B) Effects of sesamol and JNK inhibitor II on protein expression of tyrosinase in $\alpha$-MSH-treated B16F10 cells after $48 \mathrm{~h}$. Each value is presented as the mean $\pm \mathrm{SD}$. ${ }^{\# \# \#} p<0.001$ (Significant difference versus control); ${ }^{* * *} p<0.001$ (Significant difference versus $\alpha$-MSH treated group).

\section{Discussion}

Melanin synthesis is a series of oxidative reactions, and multiple enzymes are involved in these processes such as tyrosinase and TRP-1 and TRP-2. Tyrosinase, a copper-containing metalloenzyme, acts as the rate-limiting enzyme in the melanogenic pathway and plays a crucial role in initiating melanin synthesis $[23,24]$. Tyrosine is oxidized by tyrosinase to L-DOPA and DOPAquinone. DOPAquinone serves as the material to synthesize eumelanin or pheomelanin. Agents with antioxidative and tyrosinase-inhibiting properties have been previously recognized as hypopigmentation agents. Sesamol, because of its hydroxyl group, is a strong antioxidant and can inhibit biphenolase and monophenolase activity, such as that of tyrosinase, thereby inhibiting melanin synthesis in B16F10 cells [25]. A study reported that sesamol is a noncompetitive inhibitor of tyrosinase [25]. In our study, sesamol inhibited activity in both TRP-1 and tyrosinase, resulting in reduced melanin synthesis. TRP- 1 is involved in tyrosinase stabilization and is critical to eumelanin production during the melanin synthesis process. Therefore, inhibition of TRP-1 may reduce the stability of tyrosinase [26]. In this study, sesamol inhibited $\alpha$-MSH-induced melanogenesis in a dose-dependent manner. In addition, sesamol also inhibited tyrosinase activity and protein expression in B16F10 cells. These results are consistent with those of a previous study that demonstrated that at 25 and $50 \mu \mathrm{M}$, sesamol inhibited tyrosinase, TRP-1, and TRP-2 expression in melan-a cells and in melanin synthesis in zebrafish [27]. The reduction of tyrosinase, TRP-1, and TRP-2 protein levels may cause by inhibition of MITF, which is an important regulator of melanogenesis [28]. The results in this study indicate that sesamol inhibited $\alpha$-MSH-induced MITF expression, leading to inhibition of melanin biosynthesis.

To further investigate the mechanism by which sesamol inhibits melanogenesis, we examined melanin-related protein regulation in PKA/cAMP/MITF/tyrosinase, ERK/tyrosinase, and PI3K/AKT/ GSK3 $\beta /$ tyrosinase pathways. Melanin synthesis results from the following chain of events: keratinocytes secrete $\alpha$-MSH which binds to MC1R, thereby activating adenylate cyclase to synthesise cAMP, which subsequently induces phosphorylation of CREB and activation of the MITF promoter [29]. Baek and Lee investigated that sesamol inhibited cAMP level in melan-a cells at $12.5 \mu \mathrm{M}$ [27]. 
Our results indicate that sesamol inhibited $\alpha$-MSH-induced CREB and MITF expression, ultimately attenuating melanin synthesis.

To understand the regulation of signal transduction underlying the depigmenting effect, the protein inhibitors were used to examine sesamol in PKA, PI3K and MAPK pathways. In our study, melanogenesis and tyrosinase activity were inhibited in B16F10 cells after the cells were cotreated with sesamol and H-89. One study reported that inhibited MSK1 activation may disrupt the synthesis of melanin [30]. MAPK pathway modulates the transcription activity of MITF and plays important role in melanin synthesis [31,32]. Extracts of Astragalus membranaceus increased the level of ERK phosphorylation and inhibited the production of melanin in a previous study [31]. Here, reduced expression of MITF was related to inhibiting phosphorylation of ERK. It was reported that sesamol induces phosphorylation of p38 and JNK, but not ERK in melan-a cells [27]. However, our study demonstrated that sesamol inhibited $\alpha$-MSH-induced phosphorylation of ERK and, after PD 98059 cotreatment, reduced melanin content and tyrosinase activity. In addition, the results of this study indicate that sesamol's regulation of p-38 and JNK signal transduction in B16F10 cells resulted in inhibition of melanin biosynthesis. Besides MAPK pathway, PI3K/AKT and GSK3 $\beta$ expression and activity cause MITF to bind to the target sequence and induce melanogenesis. Enhancing the activity of cAMP may inhibit PI3K/AKT and GSK3 $\beta$ expression and activity [33]. In our study, sesamol elevated the expression of $p$-AKT and $p$-GSK3 $\beta$, possibly reducing MITF transcription to suppress tyrosinase gene expression and thus inhibiting melanin production and tyrosinase activity. Meanwhile, we found that through AKT and GSK3 $\beta$ activation and subsequent downregulation of MITF, CREB, tyrosinase, and TRP-1 production, sesamol inhibited $\alpha$-MSH-induced hyperpigmentation in B16F10 cells.

\section{Material and Methods}

\subsection{Chemicals and Materials}

Sesamol (purity 98\%), arbutin, L-DOPA, DL-dithiothreitol, H-89 dihydrochloride hydrate, LY294002, PD98059, and SB203580 were obtained from Sigma Chemical Co. (St. Louis, MO, USA). $\alpha$-MSH was acquired from Merck (Darmstadt, Germany). An antibody recognizing MC1R was obtained from Millipore Corporation (Billerica, MA, USA). Antibodies recognizing AKT and phospho-AKT were obtained from GeneTex, Inc. (Irvine, CA, USA). Other primary and secondary antibodies were obtained from Santa Cruz Biotechnology (Santa Cruz, CA, USA). All other chemicals and reagents used in this work were high-quality and commercially obtainable.

\subsection{Cell Cultures and Cell Viability Assay}

B16F10 melanoma cells were cultivated in Dulbecco's modified Eagle's medium (GIBCO, Invitrogen Corporation, Grand Island, NY, USA) supplemented with $10 \%$ fetal bovine serum at $37^{\circ} \mathrm{C}$ in an incubator with $5 \% \mathrm{CO}_{2}$. Cells were harvested through trypsinisation. Cell viability was measured by using the 3-(4,5-dimethylthiazol-2-yl)-2,5-diphenyltetrazolium bromide (MTT) assay, as previously described [33].

\subsection{Melanin Content and Tyrosinase Activity Assay in B16F10 Cells}

The melanin content and tyrosinase activity of B16F10 cells were measured according to a method described in previous studies [34] and using an enzyme-linked immunosorbent assay (ELISA) reader (Tecan, Grodig, Austria) at $405 \mathrm{~nm}$. Brifely, the B16F10 cells were cultured in six-well culture plates and incubated. The cells were treated with a medium containing $\alpha-\mathrm{MSH}$ and various concentrations of sesamol for $48 \mathrm{~h}$. $\mathrm{NaOH}(2 \mathrm{~N})$ was added to each well to lyse the cells, and the cells were then centrifuged. The amounts of melanin in the supernatant were spectrophotometrically measured at $405 \mathrm{~nm}$

In the tyrosinase activity assay, the B16F10 melanoma cells were plated in a 24-well plate and treated with a medium containing $\alpha$-MSH and various concentrations of sesamol for $48 \mathrm{~h}$. The medium was removed and then $1 \%$ Triton X-100 mixed in $100 \mathrm{mM}$ phosphate buffered saline was added. 
The mixture was frozen at $-80^{\circ} \mathrm{C}$ and thawed at room temperature, and then centrifuged. A freshly prepared substrate ( $15 \mathrm{mM}$ L-DOPA) was then added to the supernatant and incubated. The absorbance of each well was subsequently read.

\subsection{Western Blotting}

The expression of melanogenesis-related proteins in B16F10 cells was detected through Western blotting, as previously described $[33,35]$. Cells were cultured in a 10-cm dish overnight. Subsequently, $\alpha-\mathrm{MSH}$ and various concentrations of sesamol were added and incubated for $48 \mathrm{~h}$ or the indicated time. The protein content of cell lysates was assessed using the Bradford method. An equal amount of protein $(20 \mu \mathrm{g})$ was loaded and separated on sodium dodecyl sulfate polyacrylamide gel electrophoresis (SDS-PAGE) gel. Blots on polyvinylidene difluoride (PVDF) membranes were blocked overnight with $5 \%(w / v)$ skimmed milk solution and with the following specific antibodies: anti-actin, anti-AKT, anti- $p$-AKT, anti-CREB, anti- $p$-CREB, anti-GSK3 $\beta$, anti- $p$-GSK3 $\beta$, anti-MITF, anti-TRP-1, and anti-tyrosinase. The membranes were washed with TBST and then incubated with $G$ horseradish peroxidase. Bands were visualized using an Enhanced Chemiluminescence Plus Kit (Fujifilm, LAS-4000, Tokyo, Japan), and the density of the bands was determined using a densitometric program (MultiGauge v2.2, Fuji Photo Film Co., Tokyo, Japan).

\subsection{Statistical Analyses}

Values are presented as the mean \pm standard deviation. The results presented in this paper are representative of at least three individual experiments. Differences in the effects of various treatments were compared using the Student's t-test or ANOVA and, subsequently, Scheffe's test. $p$-values of $<0.05$ were defined as significant.

\section{Conclusion}

This study demonstrated the antimelanogenic activity of sesamol in B16F10 cells. Sesamol exhibited antimelanogenic activity through the regulation of MEK/ERK, AKT/GSK3 $\beta$, and CREB/MITF and resultant inhibition of tyrosinase and TRP-1 expression. Future applications of these findings may include use of sesamol in skin-whitening products. However, B16F10 is a rodent melanoma cell, and the results of this study have to be confirmed on human melanocytes in the future.

Acknowledgments: This study was sponsored by China Medical University (CMU103-ASIA-11) and the Ministry of Science and Technology (NSC100-2320-B-039-002-MY3; MOST104-2320-B-039-006).

Author Contributions: Po-Yuan Wu, Chien-Wei Hou, Kuo-Ching Wen, Chin-Sheng Wu, Chien-Yih Lin, and Hsiu-Mei Chiang conceived the study and contributed to study design and coordination; Ya-Jhen You and Yi-Jung Liu performed the experiments and collected the data; and Po-Yuan Wu, Kuo-Ching Wen, Chien-Yih Lin, and Hsiu-Mei Chiang wrote and revised the manuscript.

Conflicts of Interest: The authors declare no conflict of interest.

$\begin{array}{ll}\text { Abbreviations } \\ \alpha \text {-MSH } & \alpha \text {-melanocyte-stimulating hormone } \\ \text { cAMP } & \text { cyclic adenosine monophosphate } \\ \text { CAPE } & \text { caffeic acid phenethyl ester } \\ \text { CREB } & \text { cAMP response element binding protein } \\ \text { p-CREB } & \text { phospho-cAMP response element binding protein } \\ \text { DHICA } & \text { 5,6-dihydroxyindole-2-carboxylic acid } \\ \text { L-DOPA } & \text { L-dihydroxyphenylalanine } \\ \text { DMSO } & \text { dimethyl sulfoxide } \\ \text { FBS } & \text { fetal bovine serum } \\ \text { GSK3 } \beta & \text { glycogen synthase kinase } 3 \text { beta } \\ \text { MC1R } & \text { melanocortin 1 receptor }\end{array}$


MITF microphthalmia-associated transcription factor

MTT 3-(4,5-dimethylthiazol-2-yl)-2,5-diphenyltetrazolium bromide

PKA protein kinase A

TRP-1 tyrosinase-related protein-1

TRP-2 tyrosinase-related protein-2

\section{References}

1. Brenner, M.; Hearing, V.J. The protective role of melanin against UV damage in human skin. Photochem. Photobiol. 2008, 84, 539-549. [CrossRef] [PubMed]

2. D'Orazio, J.; Jarrett, S.; Amaro-Ortiz, A.; Scott, T. UV radiation and the skin. Int. J. Mol. Sci. 2013, 14, 12222-12248. [CrossRef] [PubMed]

3. Chou, T.H.; Ding, H.Y.; Lin, R.J.; Liang, J.Y.; Liang, C.H. Inhibition of melanogenesis and oxidation by protocatechuic acid from Origanum vulgare (oregano). J. Nat. Prod. 2010, 73, 1767-1774. [CrossRef] [PubMed]

4. Meira, W.V.; Heinrich, T.A.; Cadena, S.M.; Martinez, G.R. Melanogenesis inhibits respiration in B16-F10 melanoma cells whereas enhances mitochondrial cell content. Exp. Cell Res. 2016. [CrossRef] [PubMed]

5. Costin, G.E.; Hearing, V.J. Human skin pigmentation: Melanocytes modulate skin color in response to stress. FASEB J. 2007, 21, 976-994. [CrossRef] [PubMed]

6. Slominski, A.; Tobin, D.J.; Shibahara, S.; Wortsman, J. Melanin pigmentation in mammalian skin and its hormonal regulation. Physiol. Rev. 2004, 84, 1155-1228. [CrossRef] [PubMed]

7. Slominski, A.; Wortsman, J.; Luger, T.; Paus, R.; Solomon, S. Corticotropin releasing hormone and proopiomelanocortin involvement in the cutaneous response to stress. Physiol. Rev. 2000, 80, 979-1020. [CrossRef] [PubMed]

8. Hearing, V.J. Determination of melanin synthetic pathways. J. Investig. Dermatol. 2011, 131, E8-E11. [CrossRef] [PubMed]

9. D'Mello, S.A.; Finlay, G.J.; Baguley, B.C.; Askarian-Amiri, M.E. Signaling Pathways in Melanogenesis. Int. J. Mol. Sci. 2016, 17, 1144. [CrossRef] [PubMed]

10. Pillaiyar, T.; Manickam, M.; Jung, S.H. Recent development of signaling pathways inhibitors of melanogenesis. Cell. Signal. 2017, 40, 99-115. [CrossRef] [PubMed]

11. Kondo, T.; Hearing, V.J. Update on the regulation of mammalian melanocyte function and skin pigmentation. Expert Rev. Dermatol. 2011, 6, 97-108. [CrossRef] [PubMed]

12. Slominski, A.; Zmijewski, M.A.; Pawelek, J. L-tyrosine and L-dihydroxyphenylalanine as hormone-like regulators of melanocyte functions. Pigment Cell Melanoma Res. 2012, 25, 14-27. [CrossRef] [PubMed]

13. Slominski, A.T.; Zmijewski, M.A.; Zbytek, B.; Tobin, D.J.; Theoharides, T.C.; Rivier, J. Key role of CRF in the skin stress response system. Endocr. Rev. 2013, 34, 827-884. [CrossRef] [PubMed]

14. Hearing, V.J. Milestones in melanocytes/melanogenesis. J. Investig. Dermatol. 2011, 131, E1. [CrossRef] [PubMed]

15. Sun, L.; Guo, Y.; Zhang, Y.; Zhuang, Y. Antioxidant and Anti-tyrosinase Activities of Phenolic Extracts from Rape Bee Pollen and Inhibitory Melanogenesis by cAMP/MITF/TYR Pathway in B16 Mouse Melanoma Cells. Front. Pharmacol. 2017, 8, 104. [CrossRef] [PubMed]

16. Yen, F.L.; Wang, M.C.; Liang, C.J.; Ko, H.H.; Lee, C.W. Melanogenesis Inhibitor(s) from Phyla nodiflora Extract. Evid.-Based Complement. Altern. Med. 2012, 2012, 867494. [CrossRef] [PubMed]

17. Kanimozhi, P.; Prasad, N.R. Antioxidant potential of sesamol and its role on radiation-induced DNA damage in whole-body irradiated Swiss albino mice. Perumal Kanimozhi, Nagarajan Rajendra Prasad 2009, 28, $192-197$. [CrossRef] [PubMed]

18. Geetha, T.; Rohit, B.; Pal, K.I. Sesamol: An efficient antioxidant with potential therapeutic benefits. Med. Chem. 2009, 5, 367-371. [CrossRef] [PubMed]

19. Liu, Z.; Xiang, Q.; Du, L.; Song, G.; Wang, Y.; Liu, X. The interaction of sesamol with DNA and cytotoxicity, apoptosis, and localization in HepG2 cells. Food Chem. 2013, 141, 289-296. [CrossRef] [PubMed]

20. Pathak, N.; Rai, A.K.; Kumari, R.; Bhat, K.V. Value addition in sesame: A perspective on bioactive components for enhancing utility and profitability. Pharmacogn. Rev. 2014, 8, 147-155. [PubMed]

21. Yamaguchi, Y.; Hearing, V.J. Physiological factors that regulate skin pigmentation. BioFactors 2009, 35, 193-199. [CrossRef] [PubMed] 
22. Oka, M.; Nagai, H.; Ando, H.; Fukunaga, M.; Matsumura, M.; Araki, K.; Ogawa, W.; Miki, T.; Sakaue, M.; Tsukamoto, K.; et al. Regulation of melanogenesis through phosphatidylinositol 3-kinase-Akt pathway in human G361 melanoma cells. J. Investig. Dermatol. 2000, 115, 699-703. [CrossRef] [PubMed]

23. Hwang, K.S.; Yang, J.Y.; Lee, J.; Lee, Y.R.; Kim, S.S.; Kim, G.R.; Chae, J.S.; Ahn, J.H.; Shin, D.S.; Choi, T.Y.; et al. A novel anti-melanogenic agent, KDZ-001, inhibits tyrosinase enzymatic activity. J. Dermatol. Sci. 2017. [CrossRef] [PubMed]

24. Rezaei, M.; Mohammadi, H.T.; Mahdavi, A.; Shourian, M.; Ghafouri, H. Evaluation of thiazolidinone derivatives as a new class of mushroom tyrosinase inhibitors. Int. J. Biol. Macromol. 2017, 108, 205-213. [CrossRef] [PubMed]

25. Kumar, C.M.; Sathisha, U.V.; Dharmesh, S.; Rao, A.G.; Singh, S.A. Interaction of sesamol (3,4-methylenedioxyphenol) with tyrosinase and its effect on melanin synthesis. Biochimie 2011, 93, 562-569. [CrossRef] [PubMed]

26. Kobayashi, T.; Imokawa, G.; Bennett, D.C.; Hearing, V.J. Tyrosinase stabilization by Tyrp1 (the brown locus protein). J. Biol. Chem. 1998, 273, 31801-31805. [CrossRef] [PubMed]

27. Baek, S.H.; Lee, S.H. Sesamol decreases melanin biosynthesis in melanocyte cells and zebrafish: Possible involvement of MITF via the intracellular cAMP and p38/JNK signalling pathways. Exp. Dermatol. 2015, 24, 761-766. [CrossRef] [PubMed]

28. Oh, C.T.; Kwon, T.R.; Jang, Y.J.; Yoo, K.H.; Kim, B.J.; Kim, H. Inhibitory effects of Stichopus japonicus extract on melanogenesis of mouse cells via ERK phosphorylation. Mol. Med. Rep. 2017, 16, 1079-1086. [CrossRef] [PubMed]

29. Yun, C.Y.; You, S.T.; Kim, J.H.; Chung, J.H.; Han, S.B.; Shin, E.Y.; Kim, E.G. p21-Activated Kinase 4 Critically Regulates Melanogenesis via Activation of the CREB/MITF and $\beta$-Catenin/MITF Pathways. J. Investig. Dermatol. 2015. [CrossRef] [PubMed]

30. Tagashira, H.; Miyamoto, A.; Kitamura, S.; Tsubata, M.; Yamaguchi, K.; Takagaki, K.; Imokawa, G. UVB Stimulates the Expression of Endothelin B Receptor in Human Melanocytes via a Sequential Activation of the p38/MSK1/CREB/MITF Pathway Which Can Be Interrupted by a French Maritime Pine Bark Extract through a Direct Inactivation of MSK1. PLoS ONE 2015, 10, e0128678. [CrossRef] [PubMed]

31. Tsao, Y.T.; Kuo, C.Y.; Kuan, Y.D.; Lin, H.C.; Wu, L.H.; Lee, C.H. The Extracts of Astragalus membranaceus Inhibit Melanogenesis through the ERK Signaling Pathway. Int. J. Med. Sci. 2017, 14, 1049-1053. [CrossRef] [PubMed]

32. Wu, L.C.; Lin, Y.Y.; Yang, S.Y.; Weng, Y.T.; Tsai, Y.T. Antimelanogenic effect of c-phycocyanin through modulation of tyrosinase expression by upregulation of ERK and downregulation of p38 MAPK signaling pathways. J. Biomed. Sci. 2011, 18, 74. [CrossRef] [PubMed]

33. Chiang, H.M.; Chien, Y.C.; Wu, C.H.; Kuo, Y.H.; Wu, W.C.; Pan, Y.Y.; Su, Y.H.; Wen, K.C. Hydroalcoholic extract of Rhodiola rosea L. (Crassulaceae) and its hydrolysate inhibit melanogenesis in B16F0 cells by regulating the CREB/MITF/tyrosinase pathway. Food Chem. Toxicol. 2014, 65, 129-139. [CrossRef] [PubMed]

34. Kuo, Y.H.; Chen, C.C.; Lin, P.; You, Y.J.; Chiang, H.M. N-(4-bromophenethyl) Caffeamide Inhibits Melanogenesis by Regulating AKT/Glycogen Synthase Kinase 3 Beta/Microphthalmia-associated Transcription Factor and Tyrosinase-related Protein 1/Tyrosinase. Curr. Pharm. Biotechnol. 2015, 16, 1111-1119. [CrossRef] [PubMed]

35. Wen, K.C.; Chang, C.S.; Chien, Y.C.; Wang, H.W.; Wu, W.C.; Wu, C.S.; Chiang, H.M. Tyrosol and its analogues inhibit $\alpha$-melanocyte-stimulating hormone induced melanogenesis. Int. J. Mol. Sci. 2013, 14, 23420-23440. [CrossRef] [PubMed]

(C) 2018 by the authors. Licensee MDPI, Basel, Switzerland. This article is an open access article distributed under the terms and conditions of the Creative Commons Attribution (CC BY) license (http:/ / creativecommons.org/licenses/by/4.0/). 